\title{
Bohr-Sommerfeld-like quantization in the theory of walking droplets
}

\author{
J. Montes $\odot,{ }^{1,2}$ F. Revuelta $\odot,{ }^{3}$ and F. Borondo $\odot^{1,2}$ \\ ${ }^{1}$ Instituto de Ciencias Matemáticas (ICMAT), Cantoblanco, 28049 Madrid, Spain \\ ${ }^{2}$ Departamento de Química, Universidad Autónoma de Madrid, Cantoblanco, 28049 Madrid, Spain \\ ${ }^{3}$ Grupo de Sistemas Complejos, Escuela Técnica Superior de Ingeniería Agronómica, Alimentaria y de Biosistemas, \\ Universidad Politécnica de Madrid, Avda. Puerta de Hierro 2-4, 28040 Madrid, Spain
}

(Received 12 June 2019; accepted 29 April 2021; published 24 May 2021)

\begin{abstract}
Recent experiments have shown that self-propelled millimetric walking droplets bouncing on a vibrating liquid surface exhibit phenomena, such as interference or tunneling, that so far were thought to be possible only in the microscopic realm. Here we present calculations showing that the surface wave satisfies, in the long-memory limit, a Bohr-Sommerfeld quantization-like relation. This strongly suggest the possibility of a novel fundamental type of quantization in these experiments, which can simultaneously explain their emulation of the quantum behavior and, more importantly, shed light into some of the interpretational difficulties of the standard quantum theory.
\end{abstract}

DOI: 10.1103/PhysRevE.103.053110

\section{INTRODUCTION}

Wave-particle duality still remains one of the most intriguing aspects of quantum theory. Actually, particles and waves were considered apart until reunited by de Broglie in his pilot-wave, also called double-solution, theory [1,2], further elaborated by Bohm [3].

In the past few years, seminal work by Fort and Couder [4] followed by Bush [5] on a new kind of macroscopic hydrodynamic experiments [6] showed that the statistical properties of millimetric oil droplets bouncing on a surface of the same liquid excited above the so-called Faraday's threshold $\gamma_{F}$ were reminiscent of quantum experiments [7]. The collisions of such droplets, usually referred to as walking droplets (WDs) or more simply as walkers, with the surface create a wave influencing its future dynamics. Indeed, the force exerted by the surface on the WD depends on its shape, which in turn is determined by the previous history of the bounces.

Amazingly, the droplet-surface wave interaction is responsible for the emergence of a behavior that strongly resembles that of the most paradigmatic quantum phenomena. Indeed, single-particle diffraction and interference [8], single- and double-slit interference [9], tunneling [10], orbital quantization [11], and orbital level splitting [12] have been observed in those experiments. Obviously, the question of why and how this behavior arises, and if the origin is analog to that in the quantum world is an issue of paramount interest.

In this paper, we address some aspects of this question by considering the dynamics of a WD in the presence of a chaotic anharmonic coupled quartic potential. With this choice, we show that quantization in WDs does not emerge due to geometry or other dynamical peculiarity of the systems, as already observed in Refs. [13,14], but it is also a more general robust result that happens in scenarios where no invariant tori exist [15].
Our results demonstrate the emergence of quasistationary regular patterns in the underlying wave field due to coherent superposition, when a suitable Bohr-Sommerfeld (BS)-like quantization condition is fulfilled $[15,16]$. Furthermore, we show that, in the infinite memory limit, the previous condition leads to the identification of a mass-dependent constant, playing a role similar to that of the Planck's constant in quantum mechanics.

The paper is organized as follows. First, we briefly describe in the following section the fundamentals of the theory. Next, we present in Sec. III the results of our study. To sum up, we conclude this work in Sec. IV with the conclusions and the outlook.

\section{THEORY}

The dynamics of a microscopic WD bouncing on a vibrating liquid surface is customarily described (stroboscopically) using Newton's second law as [17,18]

$$
m \ddot{\mathbf{q}}\left(t^{\prime}\right)+D \dot{\mathbf{q}}\left(t^{\prime}\right)=-\nabla U(\mathbf{q})-m g \frac{A}{T_{F}} \nabla \psi_{t^{\prime}}(\mathbf{q}),
$$

where $m$ is the particle mass, $\mathbf{q}\left(t^{\prime}\right)$ its vector position at time $t^{\prime}, D$ is a friction parameter (assumed constant for simplicity), $U(\mathbf{q})$ is the external potential, $g$ is the acceleration gravity, $A$ is related to the amplitude of the wave, $T_{F}$ the oscillation period, and $\nabla \psi_{t^{\prime}}$ the gradient of the wave field. As can be seen, the last term accounts for the force exerted by the surface wave, thus being responsible for the coupling between particle and wave in this model. This wave field is taken as

$$
\psi_{t^{\prime}}(\mathbf{q})=\int_{-\infty}^{t^{\prime}} J_{0}\left[2 \pi\left|\mathbf{q}\left(t^{\prime}\right)-\mathbf{q}_{p}\left(s^{\prime}\right)\right| / \lambda_{F}^{\prime}\right] e^{-\left(t^{\prime}-s^{\prime}\right) / T_{M}} d s^{\prime},
$$

where $T_{M}$ is a characteristic time that accounts for an exponential decay of the wave field, which is proportional to $\left(1-\gamma / \gamma_{F}\right)^{-1}$, with $\gamma$ the acceleration of the bulk fluid and 
$\gamma_{F}$ Faraday's threshold. As will be shown later, Faraday's wavelength $\lambda_{F}^{\prime}$ plays here the same role as does de Broglie's wavelength, $\lambda_{\mathrm{dB}}$, in the quantum theory, and $\mathbf{q}_{p}$ gives the walker position along the "past" portion of its trajectory. In this way, Eq. (2) accounts for the history of the droplet up to time $t^{\prime}$, considering the effect of all the previous bounces on the liquid, whose surface is assumed to be deformed in space as a Bessel function of the first kind, $J_{0}$.

The corresponding dynamics is usually studied making Eq. (1) adimensional by a suitable scaling, which renders

$$
\kappa \ddot{\mathbf{x}}+\dot{\mathbf{x}}=-\nabla V(\mathbf{x})-\beta \nabla \psi_{t}(\mathbf{x}),
$$

where $t=t^{\prime} / T_{M}, \mathbf{x}(t)=\mathbf{q}(t) / \tilde{\lambda}_{F}, V(\mathbf{x})=U\left(\tilde{\lambda}_{F} \mathbf{x}\right) T_{M} /\left(D \tilde{\lambda}_{F}^{2}\right)$, $\lambda_{F}=\lambda_{F}^{\prime} / \tilde{\lambda}_{F}, \kappa=m /\left(D T_{M}\right)$, and $\beta=m g A /\left(T_{F} D\right)\left(T_{M} / \tilde{\lambda}_{F}\right)^{2}$ is a memory factor that modulates the effect of the past bounces on the wave field. Here $\tilde{\lambda}_{F}$ is a characteristic length in the system, which can be taken equal to 1 for simplicity. Despite the fact that the scaled parameters $\kappa$ and $\beta$ are related through their dependence on $D$ and $T_{M}$, and then implicitly on the $\gamma / \gamma_{F}$ ratio, we will consider hereafter these quantities as independent in order to explore the full range of possibilities that this allows.

In this work, we will consider a WD moving on a vibrating fluid surface close to the Faraday threshold, $\gamma \rightarrow \gamma_{F}$, where $T_{M} \rightarrow \infty$ and then $\beta \rightarrow \infty$. In that case, the exponential in Eq. (2) disappears, with the scaled wave field equal to

$$
\psi(x, y)=\int_{-\infty}^{t} J_{0}\left(2 \pi\left|\mathbf{x}-\mathbf{x}_{p}(s)\right| / \lambda_{F}\right) d s, \quad \mathbf{x}=(x, y) .
$$

Likewise, we consider an external potential given by the twodimensional coupled quartic oscillator

$$
V(x, y)=\frac{1}{2} x^{2} y^{2}+\frac{1}{400}\left(x^{4}+y^{4}\right),
$$

which is shown in full and desymmetrized form in the inset of Fig. 1. This potential has been extensively studied, especially in connection with quantum chaos [19-22]. The phase space associated with Eq. (5), as well as for other quartic oscillators with different parameters (see, for example, Refs. [19,2327] where rigorous results concerning orbits' stability can be found), has almost surely a mixed structure, which combines regular and irregular motion at the same energy, this producing a very rich dynamics. In particular, the motion is totally regular for the potential (5) without the coupling term $x^{2} y^{2}$, while in the absence of $x^{4}+y^{4}$ the system becomes unbounded with a very chaotic motion, which still presents some tiny regions of regularity [23]. For all practical purposes, however, these regular regions are so small that they can be neglected in general, and this is also the case for the potential (5), due to the selected values of the parameters, $1 / 2$ and $1 / 400$. As a consequence, phenomena observed in this potential are also expected in any other chaotic system due to the almost total absence of the regular structures that will result in mixed dynamics, this changing with the system. Note that a representative composite Poincare surface of section of the system, like the one shown in Fig. 1, is formed by a plethora of disconnected points densely covering the phase space, and no visible imprints of regular motion, i. e., invariant tori, are appreciated by the naked eye. Actually, the Lyapunov exponent of the generic chaotic trajectory of the

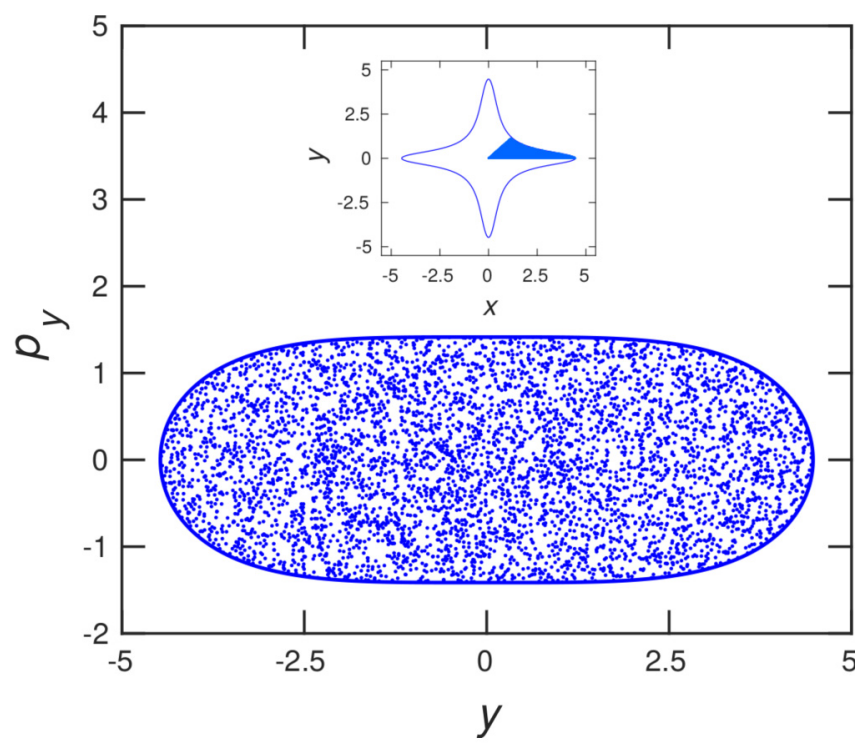

FIG. 1. Inset: Isopotential $E=1$ for the two-dimensional coupled quartic potential of Eq. (5) in the full configuration space. The blue shaded area shows the fundamental domain in the desymmetrized system, which is defined in this case for $x \geqslant y>0$. The whole potential can be constructed by reflexion of the shown fundamental domain respect to the $x$ and $y$ axes, and the diagonals $y= \pm x$. Main body: Poincaré surface of section $\left\{x=0, p_{x}<0\right\}$ for a trajectory of a unit-mass particle with energy $E=1$ moving solely under the quartic potential, a situation where no friction or particle-surface coupling effects are considered.

system shown in Fig. 1 for an energy $E$ is always positive and equal to $0.3748 E^{1 / 4}$ [21]. The choice of potential (5) ensures the inclusion of a chaotic component in the walker dynamics, even in the absence of any liquid surface. Finally, the use of a smooth potential simplifies the calculations as no boundary effects need to be taken into account [28].

In the zero-memory limit, $\beta=0$, where droplet and surface wave do not interact, and for time-independent potentials (as it is usually the case), the motion of the walkers is governed solely by the external force, $-\nabla V$, and the friction. The latter dissipates energy from the droplet until it eventually stops bouncing. The situation is quite different when the vibrating surface has an effect, i.e., when $\beta>0$. In this case, the particle sojourns the landscape formed by the mountains and valleys on the wave field also contributing, at the same time, to its shaping. If the correlation with the wave is small, i.e., when $\beta \rightarrow 0$ (low-memory limit), the particle still stops after some time due to friction [14]. On the contrary, beyond that limit the bouncing does not stop in general, since, depending on the actual value of $\beta$, the vibrating liquid surface may supply energy to the walker, this giving rise, eventually, to a very irregular and non-Markovian motion. This can be viewed as a transition from bouncing to walking, or alternatively from dissipative to self-maintained regime, in Eq. (3) [14].

\section{RESULTS AND DISCUSSION}

In this section, we report the results of our study and the corresponding discussion. For this purpose, we introduce first in Sec. III A the walker orbits that are the cornerstone for our 


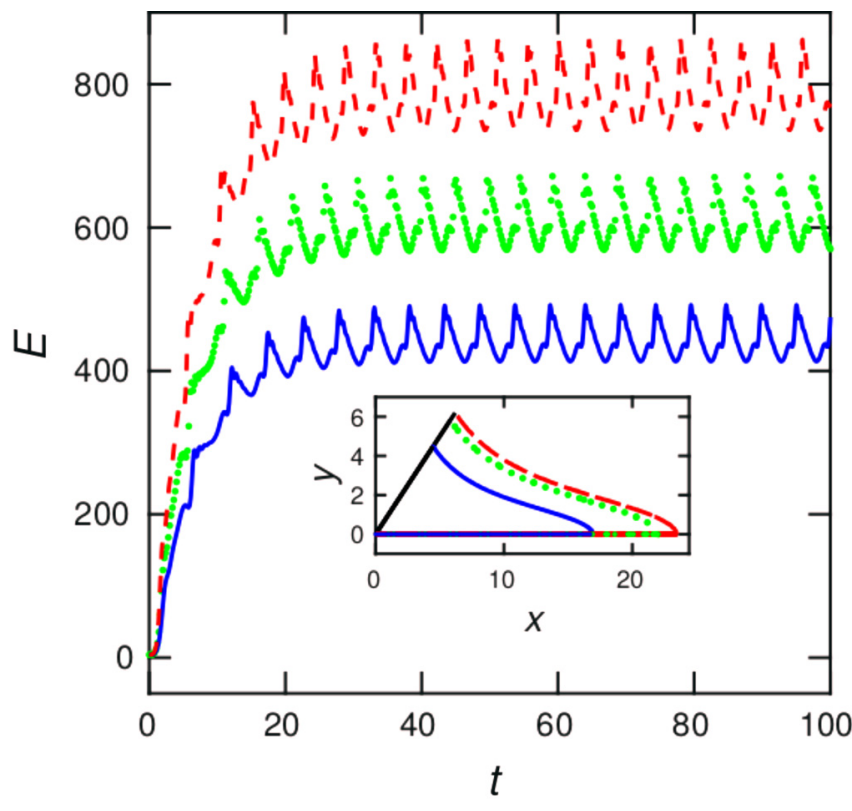

FIG. 2. Energy time evolution for the walkers that are attracted asymptotically to the horizontal periodic orbits of the coupled quartic oscillator potential (5) for $\lambda_{F}=1, \kappa=8$, and $\beta=89.59$ (blue full line), 120.00 (green dotted line), and 152.07 (red dashed line). Inset: Horizontal periodic orbits in configuration space associated with the energies shown in the main panel, and corresponding isopotential contours at $V=205.69,586.67$, and 752.73 (with the same color or line type used in the main body) for the desymmetrized system (see inset in Fig. 1).

work. Next, we present in Sec. III B the mean wave, which exhibits a quantization behavior, similar to that observed in quantum systems. Finally, we perform a systematic study of the previous quantization scheme in Sec. III C 2.

\section{A. Walker orbits}

In this paper we focus on the horizontal trajectories, three of which are shown in the inset of Fig. 2, or the alternatively symmetrically equivalent vertical ones, which have not been shown in the figure, associated with the potential (5). We have numerically verified that these periodic orbits (POs) are stable in a rather large range of parameters $(2 \leqslant \kappa \leqslant 12$ and $0 \leqslant \beta \leqslant 3000$ ). They are indeed attractors for all walker trajectories irrespective of their initial conditions, with the motion asymptotically driven to a stationary situation along the previous POs with an energy that oscillates periodically in time, as shown in the main panel of Fig. 2 [29,30]. This behavior is produced by the (periodic) driving wave field, the oscillation amplitude and period depending on the memory parameter, $\beta$. On the contrary, in the absence of correlations between particle and surface wave and without friction, i.e., in the purely classical Hamiltonian case [by setting $D=0$ and $A=0$ in Eq. (1), and then $\beta=0$ in Eq. (3)], those motions are known to be unstable POs [23]. Despite their unstable character, they still play here an important dynamical role, as at the quantum level where they give rise to the so-called "scarring" [31] of some eigenfunctions [20-22].

Figure 3 shows one of the trajectories introduced in Fig. 2 on its way to approaching the asymptotic horizontal PO
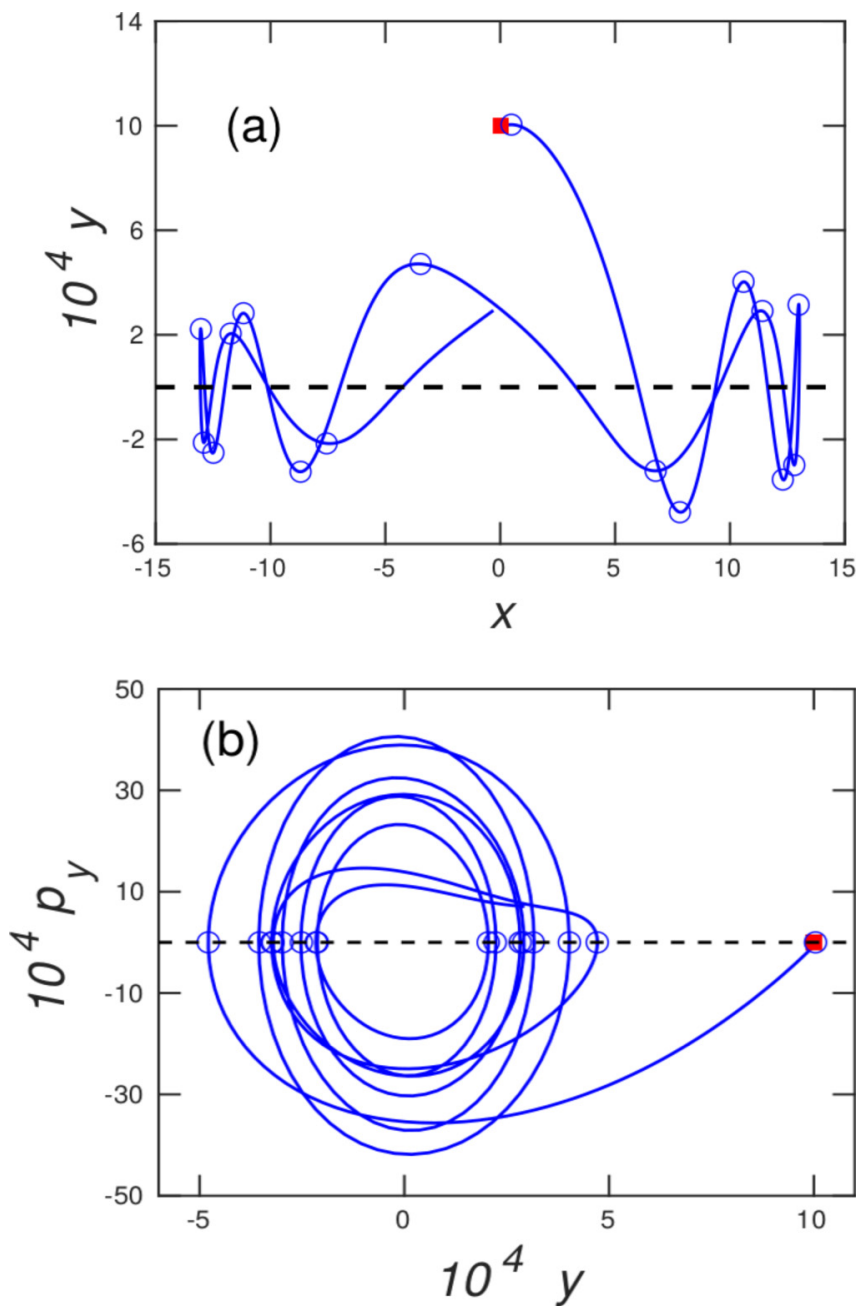

FIG. 3. (a) Configuration space $(x, y)$ and (b) transversal phase space $\left(y, p_{y}\right)$ for the walker orbit trajectory shown in blue full line in Fig. 2, which asymptotically approaches the horizontal periodic orbit defined by $\left\{y=0, p_{y}=0\right\}$. The position of the initial condition has been highlighted with a red square. The blue circles mark the positions where the traversal momentum $p_{y}$ nullifies, which is equal to the winding number ( $\mu=16$ in this case).

defined by $\left\{y=0, p_{y}=0\right\}$, both in configuration space, $(x, y)$ [Fig. 3(a)], and also in the traversal phase space [Fig. 3(b)], defined in the coordinates $\left\{y, p_{y}\right\}$ which are perpendicular to it. Note that the initial condition, which is marked as a red square, is initially separated from the horizontal PO $10^{-3}$ units of length, while the final point plotted is $\sim 5$ times closer to it. This distance reduces when considering longer time evolutions, as in Fig. 2. Similar results are also obtained for other initial conditions, as well as for other fluid parameters $(\kappa, \beta)$. However, if the initial value of $y(0)$ is too large, the motion does not end up in the horizontal but in the vertical PO $\left\{x=0, p_{x}=0\right\}$, which is dynamically equivalent to the one under study. The rate of approximation to the previous POs depends on the eigenvalues (Floquet multipliers) of the traversal monodromy matrix, which describes the (traversal) motion in its vicinity [32]. As can be seen in Fig. 3(b), the trajectory not only approaches the PO (which is here represented by the origin), as previously shown in Fig. 3(a), but it also rotates 
around it. The angle rotated when evolved over one period of time equals $16 \pi \mathrm{rad}$. As a consequence, the winding number associated with the horizontal PO equals $\mu=16$, since it is given by the number of half turns ( $\pi \mathrm{rad})$, that a surrounding trajectory describes on the transversal phase space. As the initial condition that has been considered has $p_{y}=0$, the winding number $\mu$ can be calculated by counting the number of times does $p_{y}$ nullify, something that happens at the empty circles in Fig. 3. Let us remark that the value $\mu=16$ is the same as that obtained in the Hamiltonian case, i.e., when the effect of the surface wave is absent. Let us remark that the computation of the winding number can be conducted in this case by solely counting how many times $p_{y}$ does nullify because the phase space traversal to the (horizontal) PO coincides with the Cartesian coordinates $\left\{y, p_{y}\right\}$. Other trajectories require the definition of a much more involved curvilinear set of coordinates, where the transversal plane does not coincide with any of the Cartesian axis nor does it have a constant orientation [33].

\section{B. Mean wave}

Let us discuss next the interference effects on the surface wave created by the WD motion, a point which is central in our work. To this end, we consider a mean wave defined as the properly normalized average

$$
\Psi(x, y) \sim \int_{-\infty}^{\infty} J_{0}\left(2 \pi\left|\mathbf{x}-\mathbf{x}_{p}(s)\right| / \lambda_{F}\right) d s, \quad \mathbf{x}=(x, y),
$$

which corresponds to the infinite long-time limit of Eq. (4) adequately normalized. This function can also be obtained as the convolution of $J_{0}$ with the statistical distribution of the droplet position [13]. The accuracy of the mean wave was verified in the Ref. [34], where it was demonstrated its ability to reproduce the results obtained for "quantum corrals" [35]. In that work, time averages of the instantaneous wave field over a time of $\sim 230 T_{M}$ were conducted. In our case, those (long-time) averages have been conducted over a lapse of time 6.5 times larger. Nonetheless, this difference is not significant, as the shape of the time average of the instantaneous wave field remains almost unaffected when computed over times larger than a critical threshold.

If the droplet dynamics is irregular, the wave field interacts destructively with itself, thus yielding an approximate homogeneous low-intensity pattern à la Shnirelman [36,37], with no recognizable features in it. Contrary, if the WD moves periodically in time, inducing a periodic field $\psi_{t}$, as in the present case, the correlations implicit in Eqs. (1)-(4) may give rise to coherence leading to the emergence of regular patterns in $\Psi$ in Eq. (6). Notice that this must not necessarily always be the case, since for a constructive interference to exist extra conditions must be fulfilled. For example, in the semiclassical theory [16] quantization conditions appear for the action in terms of a universal (Planck) constant [15].

To illustrate the previous point in our case, we present in Fig. 4 the results for $|\Psi(x, 0)|^{2}$ obtained for the trajectories and parameters depicted in Fig. 2 (and using the same color code), along with the results for other trajectories with different memory parameters (in pink). Several comments are in order. First, in all cases the mean wave accumulates in an

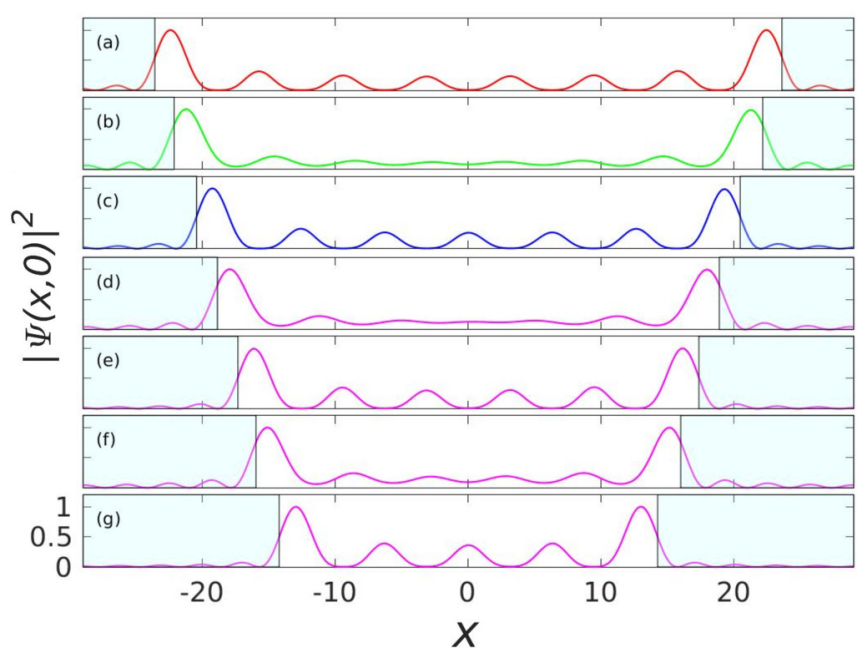

FIG. 4. Mean wave defined as the time average of the instant surface wave in Eq. (4) for $\lambda_{F}=1, \kappa=8$, and $\beta=152.07$ (a), 120.00 (b), 89.59 (c), 67.00 (d), 49.412 (e), 37.00 (f), and 24.94 (g). The position of the turning point, $x_{ \pm}^{\mathrm{TP}}$, has been marked as a vertical line, which separates the classically allowed (central white) from the forbidden region (blue shaded).

"inner" region, bounded by the two prominent outer global maxima located at $x_{ \pm}^{\mathrm{WD}, \max }$ which are near, although not exactly at the walker orbit turning points, $x_{ \pm}^{\mathrm{TP}}$, their value outside this region always being very small. The origin of these results lie on the fact that the WD motions create a wave that is always confined within that inner region. Accordingly, the WD spends more time in the vicinity of the turning points, thus building up more probability in their neighborhoods, as happens when semiclassically constructing quantum wave functions. Note also in Fig. 4 that a small part of the mean wave goes beyond the position of the turning points entering the classically forbidden region (outmost shaded), similar to what happens in the quantum case. Again, the two previous facts indicate that the WD model exhibits similarities to wellknown quantum effects, as pointed out in the Introduction.

Second, notice that Figs. 4(a), 4(c), 4(e), and 4(g), which show the mean waves for $\beta=152.07,89.59,49.41,24.94$, respectively, exhibit a clear nodal pattern, which is obviously the result of a constructive interference produced on $\psi_{t}$ as the walker progresses along its trajectory. Moreover, the number of nodes increases with the value of $\beta$ from one panel to the next by one unit, from 4 to 7 in this case.

Third, notice that the waves for the intermediate values of $\beta=120.00,67.00$, and 37.00 shown in Figs. 4(b), 4(d), and 4(f), respectively do not have, on the contrary, such a clear set of nodes, which indicates that the interference there is destructive. The emergence or absence of a well-defined set of nodes depends strongly on the position of the turning points of the WD, which are also responsible of the position of the nodes in the Bessel function present in the integral (6) [29]. In order to demonstrate this fact, we show in Figs. 5(a)$5(\mathrm{~g})$ two Bessel functions centered at the WD-turning points, $J_{0}\left(2 \pi\left|x-x_{ \pm}^{\mathrm{TP}}\right| / \lambda_{F}\right)$ and in Figs. 5(h)-5(n) their sum. As can be seen, while in Figs. 5(h), 5(j), 5(1), and 5(n) the distances between the turning points are such that the nodes of both 

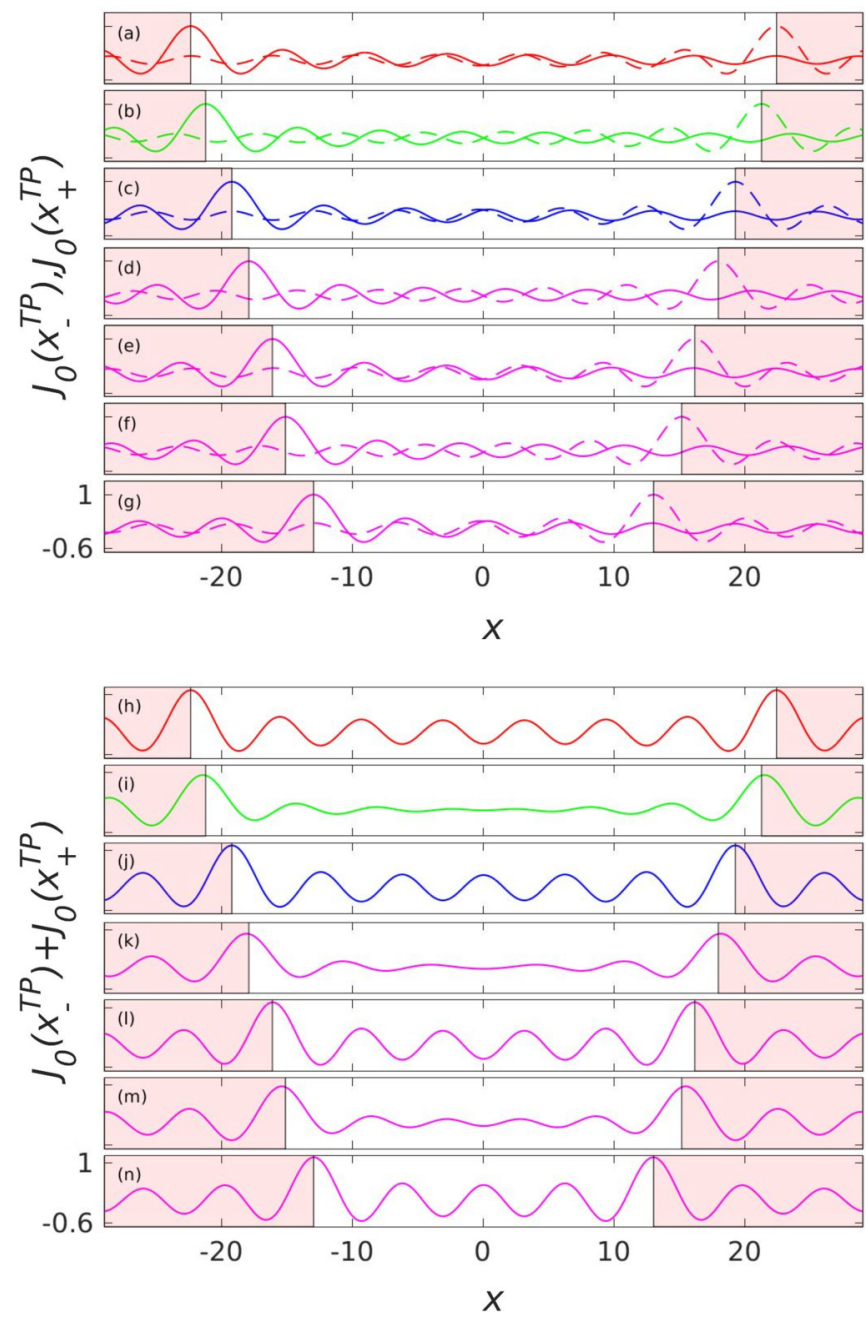

FIG. 5. (a)-(g) Bessel functions centered at the turning points of the walking droplet, $x_{-}^{\mathrm{TP}}$ (continuous lines), and $x_{+}^{\mathrm{TP}}$ (dashed lines), and their sums (h)-(m). Parameters and coloring as in Fig. 4.

Bessel functions almost coincide, for the separation distances shown in Figs. 5(i), 5(k), and 5(m) the nodes do not overlap [38]. The emergence of the clear nodal pattern visible in Figs. 5(h), 5(j), 5(l), and 5(n) is then caused by the constructive interference of the two separated Bessel functions. A similar behavior is also observed in Figs. 4(a), 4(c), 4(e), and $4(\mathrm{~g})$, whose origin must then also be the constructive interference of the instantaneous wave that drives the droplet, as heuristically shown in Ref. [29]; such a nodal pattern in the mean wave is reminiscent of the quantization that is observed in quantum systems. Contrary, the interference between the two separated Bessel functions in Figs. 5(i), 5(k), and 5(m), of the instantaneous wave with itself in Figs. 4(b), 4(d), and 4(f) must be destructive.

Fourth, the situation described in the two previous points is highly reminiscent of what happens in quantum mechanics.

Fifth, let us conclude this section by pointing out that similar results are obtained for other values of $\kappa$, since this parameter changes only the value of the $\beta$ parameter where quantization is observed (see discussion of Fig. 7 below). Actually, the minimum number of nodes that is observed, something that happens for the smallest value of $\beta$, may change from one $\kappa$ to the other.

\section{Quantization of walking droplets}

Let us investigate next the condition for the emergence of the constructive interference responsible for the results of Fig. 4 and make use of it to be able to reproduce the nodal structure of the quantum eigenfunctions. In this respect, it should be first noticed that, although quantization effects are not uncommon in wave phenomena, the peculiarity here is that our mean wave is strongly linked to the WD motion [39]. For this purpose, we have divided this section in two parts. First, we present in Sec. III C 1 the fundamental ansatz of this work, namely, that those mean waves (6) which have a clear nodal pattern satisfy the BS quantization condition. Second, we address in Sec. III C 2 which conditions are required in order to satisfy the previous equation for a constant $\hbar$.

\section{Quantization condition}

Guided by the results in the WD experiments [8-12], we will restrict our research goal to check only if the quantized mean waves shown in Fig. 4 fulfill a quantization condition similar to those satisfied by the semiclassical approximations. According to the semiclassical theory, the energy of the quantum eigenfunctions fulfill the well-known BS quantization

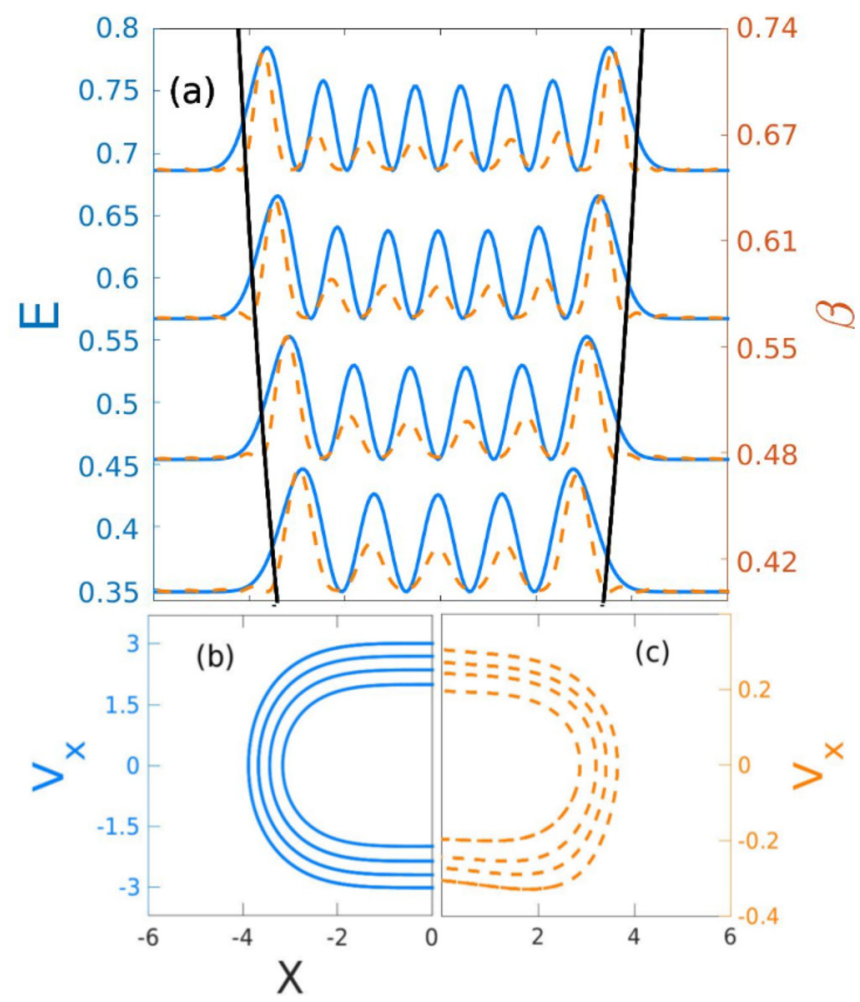

FIG. 6. (a) (Squared) Quantum eigenfunctions (blue solid line) for the one-dimensional quartic potential $V(x, 0)$ of Eq. (5) (black line) and (squared) mean wave (6) for $V(x, y)$ projected in the $x$ axis (orange dashed line), for $n_{x}=4-7$. The energy and $\beta$ scales are given at the left and right vertical axes, respectively. Corresponding phase space pictures obtained from (b) purely classical and (c) walking droplet trajectories. 
TABLE I. Protocol for the different tasks and involved unknowns calculated in each step of the determination of the value of the quantization constant of a walking droplet, as described in Secs. III B and III C 2.

\begin{tabular}{|c|c|c|}
\hline Step & Task & Unknown \\
\hline \multicolumn{3}{|c|}{ QUANTUM } \\
\hline 1 & Computation of the quantum eigenfunctions for a given reduced mass, $\kappa$ & $\psi_{n}$ \\
\hline 2 & Determination of the number of nodes of $\psi_{n}^{2}$ & $n$ \\
\hline 3 & For each $n$, determination of the position of the largest maxima of $\psi_{n}^{2}$ & $x_{ \pm}^{\mathrm{Q}, \max }$ \\
\hline 4 & $\begin{array}{l}\text { WALKING DROPLET } \\
\text { Selection of the adequate Bessel function centered at } x_{-}^{\mathrm{Q}, \max } \text { such that } \\
\text { it has a maximum at } x_{+}^{\mathrm{Q}, \max } \text { and } n \text { nodes within the range }\left(x_{-}^{\mathrm{Q}, \max }, x_{+}^{\mathrm{Q}, \max }\right)\end{array}$ & $\lambda_{F}$ \\
\hline 5 & $\begin{array}{l}\text { Calculation of the memory factor } \beta \text { such that the maxima of the } \\
\text { mean wave, } x_{ \pm}^{\mathrm{WD}, \max } \text {, coincide with } x_{ \pm}^{\mathrm{Q}, \max }\end{array}$ & $\beta, \mathbf{x}, \Psi$ \\
\hline & SEMICLASSICAL BOHR-SOMMERFELD CONDITION & \\
\hline 6 & Computation of the parameters of the walking droplet & $\oint p_{x} d x, \mu$ \\
\hline 7 & Calculation of the hydrodynamical quantization constant that fulfills Eq. (7) & $\hbar_{\beta}^{\mathrm{WD}}$ \\
\hline
\end{tabular}

condition [15] for the phase, mechanical plus topological [33],

$$
\frac{1}{\hbar} \oint p_{x} d x-\frac{\mu \pi}{2}=2 \pi n, \quad n=0,1,2, \ldots
$$

We will apply Eq. (7) to our case, taking into account that the constructive interference $[40,41]$ is observed only for certain values of the action.

In this work, it is assumed that the WD mean waves also fulfill Eq. (7); then the existence of a hydrodynamical constant $\hbar^{\mathrm{WD}}$ is simply postulated. In the quantum case, Eq. (7) implies that for a time-independent potential, like the one given by Eq. (5), only certain energies (those which yield the adequate action) are allowed, at least if the system is bounded. However, in the WD problem the energy is time-varying, and, as a consequence, the energy is not well defined. Then it is the memory $\beta$ the parameter that takes over the role played by the energy in a quantum system. Still, a direct comparison between the mean waves (6) presented in Fig. 4 and the corresponding quantum eigenfunctions obtained for the same reduced mass $\kappa$ [cf. continuous lines in Fig. 6(a)] cannot be performed, since they appear at different spatial ranges. To circumvent this problem, we conduct the procedure described in the next section.

\section{Quantization constant in walking droplets}

The procedure to calculate a quantization constant associated with the WD is briefly summarized in Table I. It consists of seven steps, which combine some quantum computations (steps 1-3) with others in the WD system (steps 4 and 5), and ends up with two final points (steps 6 and 7), where the hydrodynamical constant is calculated.

To begin, the first three steps are devoted to the computation of the quantum eigenfunctions, $\psi_{n}$, which are taken as the reference. These steps are necessary in order to establish the location of the outer maxima, $x_{ \pm}^{\mathrm{Q}, \max }$, in the probability density associated with the $n$th energy level, which has $n$ nodes within the range $x \in\left(x_{-}^{\mathrm{Q}, \max }, x_{+}^{\mathrm{Q}, \max }\right), n$ also being the integer fulfilling the quantization condition (7).

Steps 4 and 5 are the cornerstone of our procedure, since they refer to the calculations for the WD. Here optimal $\lambda_{F}$ and $\beta$ parameters are computed in order to be able to compare with the quantum function, $\psi_{n}$, calculated in the previous three steps. Following the approach reported in Ref. [29], step 4 is devoted to the calculation of the best suited value of $\lambda_{F}$. For this purpose, two Bessel functions, $J_{0}\left(\left|x-x_{ \pm}^{\mathrm{Q}_{\max }}\right| / \lambda_{F}\right)$, are centered at the points $x_{ \pm}^{\mathrm{Q}, \max }$ such that their sum has the best defined $n$ nodes (being $n$ the integer taken as reference in step 2) within the range $x \in\left(x_{-}^{\mathrm{Q}, \max }, x_{+}^{\mathrm{Q} \text {,max }}\right)$; in this situation, the nodes of each individual Bessel function are almost coincident (see discussion of Fig. 5 above). This is done by selecting the $\lambda_{F}$ value such that the Bessel function $J_{0}\left(\left|x-x_{ \pm}^{\mathrm{Q}_{\max }}\right| / \lambda_{F}\right)$ has $n$ nodes within the range $x \in\left(x_{-}^{\mathrm{Q}, \max }, x_{+}^{\mathrm{Q} \text {,max }}\right)$ and a maximum at $x_{ \pm}^{\mathrm{Q}, \max }$. Subsequently, the optimal value of $\beta$ is calculated, such that the location of the maxima of the corresponding mean wave (6), $x_{ \pm}^{\mathrm{WD}, \max }$, coincides with those of the quantum eigenfunctions, $x_{ \pm}^{\mathrm{Q}, \max }$, previously calculated in step 3 . Note that the mean wave also has $n$ nodes withing the WD turning points. Tuning the value of $\lambda_{F}$ adds an additional degree of freedom to the quantization phenomenon, as it changes the distances between the nodes of oscillations of the Bessel functions. This simple approach neglects, however, a fundamental point in relation with Eq. (7), namely, the dependence existing between $\lambda_{F}$ and $\beta$, which plays here an analogous role to that existing between $\lambda_{\mathrm{dB}}$ and $E$ in the quantum theory, i.e., the renowned de Broglie's principle. Some interesting results on this dependence have been reported in Ref. [42].

Finally, steps 6 and 7 deal with the calculation of the effective hydrodynamical constant, $\hbar_{\beta}^{\text {WD }}$. For this purpose, one must calculate in step 6 the classical action and the winding number associated with the PO of the WD, and include those values (as well as the reference integer, $n$ ) in Eq. (7) in order to calculate the value of the quantization constant, which depends in general on the values of $n$ and $\beta$.

Figure 6 shows some of the results that are obtained. Here we have used as a reference the quantum results obtained from the potential (5) in the limit of $y$ very small, which makes the system separable. The eigenfunctions associated with the quantum numbers $n_{x}=4,5,6,7$ and $n_{y}=0$ are shown at their corresponding energies in Fig. 6(a) in blue. These results have been obtained by solving the Schrödinger equation associated with the effective one-dimensional potential $V(x, 0)$, by setting $y=0$ in Eq. (5), using a basis set formed by $\sim 700$ ele- 
ments of the discrete variable representation (DVR) [43]. We have chosen to make this comparison since, for sufficiently long times, the motion of the WD takes place in the immediate neighborhood of the $x$ axis (see Fig. 3), and then the influence of the transversal degree of freedom $y$ is negligible [44]. The mean waves yielded by the procedure described above appear superimposed in the orange dashed line, being the scale for $\beta$ shown at the right vertical axis of the plot. The absolute maxima of the WD and quantum functions are matched with a precision of 0.05 in our case, a value that is high enough for our purposes. Nonetheless, this precision can be arbitrarily increased up to machine precision if necessary. A more subtle aspect is the agreement between the inner maxima of the quantum function and the quantized mean wave. In this case, there is no way to fit the position of the set of maxima of the mean wave with that of the quantum eigenfunction. Then the distance between the relative maxima of the WD and the quantum functions found in the "inner" region emerges as an excellent indicator of the accuracy of our calculations. In our case, this difference is less than 0.08 . Notice also that the intensity of the mean waves in the inner region is always much smaller than the corresponding quantum counterpart.

Finally, the crucial step in our discussion on the quantization of the WD theory is to confirm that the underlying WD motion, corresponding to our "constructively interfering" mean waves, fulfill a BS-like quantization condition equivalent to Eq. (7), as happens in the quantum case. For this purpose, we present in Fig. 6(b) the phase space pictures for the horizontal PO (left) and walker orbits (right), corresponding to the wave functions in Fig. 6(a). The former are propagated by numerically integrating Hamilton's equations of motion at the quantum eigenenergies: $E_{4}, E_{5}, E_{6}$, and $E_{7}$. The latter are obtained using Eq. (3) and the values of $\lambda_{F}$ and $\beta$ obtained with the procedure described above. Notice the difference between the two plots: while the first one is symmetric with respect to both $x=0$ and $v_{x}=0$, the second is symmetric with respect to the point $x=v_{x}=0$. From these plots, the values for the mechanical action to be used in (7) are obtained. Regarding the topological phase, the winding number $\mu$ is not always easy to ascertain. For unstable POs it coincides with the Maslov index [45], but in general it can be numerically computed as described in Ref. [46].

Once the total actions are known, values for the quantization parameter in the macroscopic hydrodynamical WD theory, $\hbar_{\beta}^{\mathrm{WD}}$, can be obtained from Eq. (7). If the ansatz at the beginning of this section is coherent, $\hbar_{\beta}^{\mathrm{WD}}$ will be constant, this proving that the emergence of the constructive interference in the mean wave has a physical connection to the walker motion, equivalent to what that existing in the quantum theory between the de Broglie's wave and the particle motion, and moreover it has the same origin. The corresponding results are shown in Fig. 7 for a large number of values of the memory parameter $\beta$ (or $n$ ) and the scaled mass $\kappa$. As can be seen, the resulting values of $\hbar_{\beta}^{W D}$ for each value of $\kappa$ are not constant. However, they tend to be so in the large memory limit, where the different curves converge to a well-defined asymptotic value $\hbar_{\infty}^{\mathrm{WD}}$. The corresponding values, obtained by fitting the data to the expression $\hbar_{\beta}^{\mathrm{WD}}=\hbar_{\infty}^{\mathrm{WD}}[1-a /(\beta-b)]$, are presented in the inset in Fig. 7 , where they are seen to follow the power law $\hbar_{\infty}^{\mathrm{WD}}=0.56 \kappa^{0.13}$.

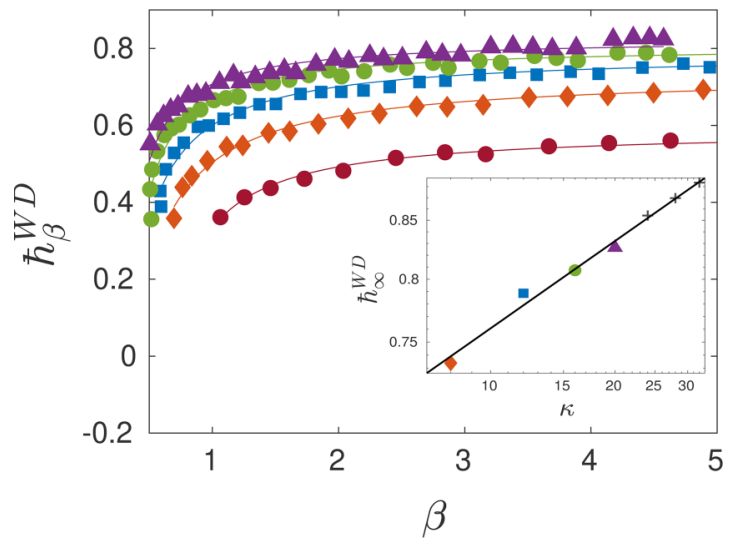

FIG. 7. Quantization constant, $\hbar_{\beta}^{W D}$, obtained from a BohrSommerfeld-like expression (7) as a function of the memory parameter $\beta$ for different values of the scaled mass $\kappa: 4$ (red circles), 8 (yellow diamonds), 12 (blue squares), 16 (green crosses), and 20 (magenta plus signs), respectively. The dependence of the asymptotic values, $\hbar_{\infty}^{W D}$, with $\kappa$ is shown in the inset.

The existence of the hydrodynamic constant defined in this work can be tested in experiments, for example, using a chaotic billiard such as the Bunimovich stadium [37]. In this respect the scaled mass $\kappa$ in a typical experiment is usually one order of magnitude smaller than the parameters used in this work. Moreover, for the set of parameters selected here, getting sufficiently large values of $\beta$ would require values $\gamma / \gamma_{F}$ larger than $\sim 0.96$. Still, the asymptotic behavior inferred for large memory $\beta$, and then for the long-memory limit, for all the results presented in Fig. 7 makes us expect similar results also for that range of parameters. A more involved aspect is the dependence that exists between $\beta$ and $\kappa$ through the ratio $\gamma / \gamma_{F}$. Reaching the long-memory limit in an experiment by getting closer to the Faraday threshold $\left(\gamma \rightarrow \gamma_{F}\right)$, would reduce the value of $\kappa$, which tends to zero proportionally to $\left(1-\gamma / \gamma_{F}\right)$, and increase the memory parameter, which diverges with $\left(1-\gamma / \gamma_{F}\right)^{-2}$, and then no constant value of $\hbar_{\beta}^{\mathrm{WD}}$ would be observed. Tuning the oscillation period would be an alternative to change the memory factor $\beta$ while keeping constant the scaled mass $\kappa$ in order to identify $\hbar_{\infty}^{\mathrm{WD}}$.

\section{CONCLUSIONS AND OUTLOOK}

Summing up, in this paper we have studied the dynamics of a WD in the presence of a two-dimensional (chaotic) quartic oscillator, demonstrating the emergence at specific values of the memory parameter $\beta$ of (averaged) mean waves created by the walker which exhibit clear nodal patterns, produced by the coherent interference in the instant wave field, similar to that appearing in microscopic quantum systems. That is, the memory $\beta$ is the tuning parameter that determines the quantization condition of the system.

Furthermore, by computing the mechanical action and winding number of the walker orbits, and the number of nodes of the mean wave, we have been able to identify a quantization condition which is completely similar to the usual semiclassical BS condition. More importantly, we have demonstrated that the associated quantization parameter, playing the role 
of the universal quantum Planck's constant, remains fairly constant for sufficiently large values of the memory.

\section{ACKNOWLEDGMENTS}

This work has been partially supported by the Spanish Ministry of Science, Innovation and Universities, Gob- ierno de España under Contracts No. PGC2018-093854BI00 and Severo Ochoa SEV-2015-0554, and by the Comunidad de Madrid under project APOYO-JOVENES4L2UB6-53-29443N (GeoCoSiM), financed within the Plurianual Agreement with the Universidad Politécnica de Madrid in the line to strengthen the research of young doctors.
[1] L. de Broglie, Nature (London) 112, 540 (1923).

[2] L. de Broglie, A. Knodel, and J. C. Miller, Non-linear Wave Mechanics: A Causal Interpretation (Elsevier, Amsterdam, 1960).

[3] D. Bohm, Phys. Rev. 85, 166 (1952).

[4] Y. Couder, S. Protiére, E. Fort, and A. Boudaoud, Nature (London) 437, 208 (2008).

[5] J. W. M. Bush, Phys. Today 68(8), 47 (2015).

[6] J. W. M. Bush and A. U. Oza, Rep. Prog. Phys. 84, 017001 (2021).

[7] M. Faraday, Phil. Trans. Royal Soc. (London) 121, 299 (1831).

[8] Y. Couder and E. Fort, Phys. Rev. Lett. 97, 154101 (2006).

[9] G. Pucci, D. M. Harris, L. M. Faria, and J. W. M. Bush, J. Fluid Mech. 835, 1136 (2018).

[10] A. Eddi, E. Fort, F. Moisy, and Y. Couder, Phys. Rev. Lett. 102, 240401 (2009).

[11] E. Fort, A. Eddi, A. Boudaoud, J. Moukhtar, and Y. Couder, Proc. Nat. Acad. Sci. USA 107, 17515 (2010).

[12] A. Eddi, J. Moukhtar, S. Perrard, E. Fort, and Y. Couder, Phys. Rev. Lett. 108, 264503 (2012).

[13] M. Durey, P. A. Milewski, and J. W. M. Bush, Chaos 28, 096108 (2018).

[14] A. U. Oza, A trajectory equation for walking droplets: Hydrodynamics pilot-wave theory, Ph.D. thesis, MIT, Cambridge, MA, 2014.

[15] A. D. Stone, Phys. Today 58(8), 37 (2005).

[16] M. Brack and R. Bhaduri, Semiclassical Physics (AddisonWesley, New York, 1997).

[17] S. E. Turton, M. M. P. Couchman, and J. W. M. Bush, Chaos 28, 096111 (2018).

[18] R. Dubertrand, M. Hubert, P. Schlagheck, N. Vandewalle, T. Bastin, and J. Martin, New J. Phys. 18, 113037 (2016).

[19] O. Bohigas, S. Tomsovic, and D. Ullmo, Phys. Rep. 223, 43 (1993).

[20] G. G. de Polavieja, F. Borondo, and R. M. Benito, Phys. Rev. Lett. 73, 1613 (1994).

[21] F. Revuelta, R. M. Benito, F. Borondo, and E. Vergini, Phys. Rev. E 87, 042921 (2013).

[22] F. Revuelta, E. Vergini, R. M. Benito, and F. Borondo, Phys. Rev. E 102, 042210 (2020).

[23] P. Dahlqvist and G. Russberg, Phys. Rev. Lett. 65, 2837 (1990).

[24] T. Kapela and C. Simó, Nonlinearity 30, 965 (2017).

[25] R. L. Waterland, J.-M. Yuan, C. C. Martens, R. E. Gillilan, and W. P. Reinhardt, Phys. Rev. Lett. 61, 2733 (1988).

[26] B. Eckhardt, G. Hose, and E. Pollak, Phys. Rev. A 39, 3776 (1989).

[27] A. Lakshminarayan, M. S. Santhanam, and V. B. Sheorey, Phys. Rev. Lett. 76, 396 (1996).
[28] T. Gilet, Phys. Rev. E 93, 042202 (2016).

[29] J. Montes, F. Revuelta, and F. Borondo, J. Phys. Chem. A 123, 1622 (2019).

[30] B. Filoux, M. Hubert, P. Schlagheck, and N. Vandewalle, Phys. Rev. Fluids 2, 013601 (2017).

[31] E. J. Heller, Phys. Rev. Lett. 53, 1515 (1984).

[32] P. Cvitanović, R. Artuso, R. Mainieri, G. Tanner, and G. Vattay, Chaos: Classical and Quantum (Niels Bohr Institute, Copenhagen, 2016).

[33] B. Eckhardt and D. Wintgen, J. Phys. A 24, 4335 (1991).

[34] D. J. Sáenz, T. Cristea-Platon, and J. W. M. Bush, Nat. Phys. 14, 315 (2018).

[35] M. F. Crommie, C. P. Lutz, and D. M. Eigler, Science 262, 218 (1993).

[36] A. I. Shnirelman, Ups. Mat. Nauk 29, 181 (1974).

[37] G. Casati and B. Chirikov, Quantum Chaos between Order and Disorder (Cambridge University Press, Cambridge, 1995).

[38] Bessel functions $J_{0}$ are not exactly periodic, and then it not possible to perfectly adjust the oscillations of one of them centered at $x_{+}^{\mathrm{TP}}$ with another one centered at $x_{-}^{\mathrm{TP}}$. However, the difference of the width of each oscillation does not change significantly, and then it can be taken in practice as constant, as the distance between two consecutive nodes of a Bessel function rapidly converges to $\pi$. Then the error when approximating it as a constant always remains below $2 \%$. For example, the distance between the two first zeros of the Bessel function $J_{0}$ equals $\sim 1.01346 \pi$, while the distance between the 11 th and 10 th zeros is $\sim 1.00033 \pi$, and the distance between the 1001th and 1000th zeros is $\sim 1.000000038 \pi$.

[39] M. Hatifi, R. Willox, S. Colin, and T. Durt, Entropy 20, 780 (2018).

[40] S. Perrard, M. Labousse, M. Miskin, E. Fort, and Y. Couder, Nat. Commun. 5, 3219 (2014).

[41] M. Labousse, S. Perrard, Y. Couder, and E. Fort, New J. Phys. 16, 113027 (2014).

[42] L. Tadrist, J.-B. Shim, T. Gilet, and P. Schlagheck, J. Fluid Mech. 848, 906 (2018).

[43] Z. Bacic and J. C. Light, Ann. Rev. Phys. Chem. 40, 469 (1989).

[44] The $x$ and $y$ degrees of freedom already differ by four orders of magnitude in Fig. 3(a), a value that increases with time by one every 10 periods due to the approximation $(y \rightarrow 0)$ to the horizontal PO.

[45] S. C. Creagh, J. M. Robbins, and R. G. Littlejohn, Phys. Rev. A 42, 1907 (1990).

[46] F. Revuelta, E. Vergini, R. M. Benito, and F. Borondo, J. Phys. Chem. A 120, 4928 (2016). 